\title{
A pressure-based single beat method for estimation of right ventricular ejection fraction: proof of concept
}

\author{
To the Editor:
}

The utility of considering right ventricular (RV) contractility and afterload as independent entities and summarising their balance or "coupling" using single beat methods has become widely appreciated [1-3]. Typically expressed as the ratio of end-systolic ventricular elastance (Ees, a load-independent measure of contractility), to arterial elastance (Ea, a lumped parameter measure of afterload) data suggest that when Ees/Ea reaches a critical threshold, the risk of cardiovascular decompensation begins to rise [4].

Pressure-based single beat methods have two central features: prediction of Pmax, the theoretical pressure generated within the RV if contraction remained isovolumic, and definition of end-systolic pressure (ESP). These variables are then used to calculate Ees as (Pmax-ESP)/SV, and Ea as ESP/SV, where SV is the stroke volume. However, it remains unclear what a "normal" RV Ees/Ea value is, due in part to variation in how ESP is defined $[5,6]$, a consideration highlighted in a recent clinical study comparing single and multi-beat determination of Ees/Ea [7]. Additionally, directly relating Ees/Ea to right ventricular ejection fraction (RVEF), a variable clinicians are more familiar with, is challenging. RVEF has been repeatedly shown to predict outcomes in patients with severe pulmonary hypertension $(\mathrm{PH})$ [8], and while cardiac magnetic resonance imaging (cMRI) or three-dimensional echocardiography allow for direct measurement of RVEF, they are not routinely used for repeated measurement of RVEF during a clinically indicated right heart study. The present proof of concept study was designed to test the hypothesis that a method using readily available software and based entirely on analysis of the right ventricular pressure (RVP) waveform can effectively track acute changes in RVEF.

Archived measurements of RVP and RV volume provided by conductance/micromanometer catheter were retrospectively analysed. Data had been acquired from 15 anaesthetised swine $(\sim 55 \mathrm{~kg})$ under Institutional Animal Care and Use Committee-approved protocols and in accordance with the National Institutes of Health Guide for the Care and Use of Laboratory Animals. Prospective clinical data were acquired under a protocol approved by the Yale Human Investigation Committee. Input signals were sampled at $200 \mathrm{~Hz}$ and measured reference values for RVEF calculated from beat-to-beat RV volume as SV/end-diastolic volume. Experimental data had been recorded before and during interventions to alter RV afterload alone or in combination with inotropic depression or augmentation.

The Dynamic Fit Wizard within SigmaPlot (version 13, Systat Software, Inc., San Jose, CA) was used to predict Pmax with a distribution function (the 4 parameter Weibull peak fit). In a pilot study involving 15 RVP waveforms with peak pressures ranging from $\sim 20$ to $50 \mathrm{mmHg}$, the distribution function was found to yield Pmax values that were within $3 \pm 7 \mathrm{mmHg}$ of those derived using a more conventional sinusoidal function (figure 1a). For ejection fraction estimation, an alternative approach for defining the RVP segments used in a Pmax prediction model was applied [9]. In addition, ESP was defined by approximating the point of maximal time varying RV elastance [10] using the second derivative of RVP (figure 1b). Ejection fraction was then estimated as Ees/(Ees+Ea) (figure 1c). Since SV is a common factor in the calculation of both Ees and Ea, this equation can be reduced to (Pmax-ESP)/Pmax or further simplified to $1-(\mathrm{ESP} / \mathrm{Pmax})$.

From the waveform library, a dataset containing 69 individual RVEF measurements was constructed and paired with RVEF estimates over the same sampling interval. Method comparison procedures were applied

@ERSpublications

This proof of concept study suggests that estimation of RV ejection fraction based solely on analysis of the RV pressure waveform may be feasible http://bit.ly/32ZRoOi

Cite this article as: Heerdt PM, Kheyfets V, Charania S, et al. A pressure-based single beat method for estimation of right ventricular ejection fraction: proof of concept. Eur Respir J 2020; 55: 1901635 [https:// doi.org/10.1183/13993003.01635-2019]. 


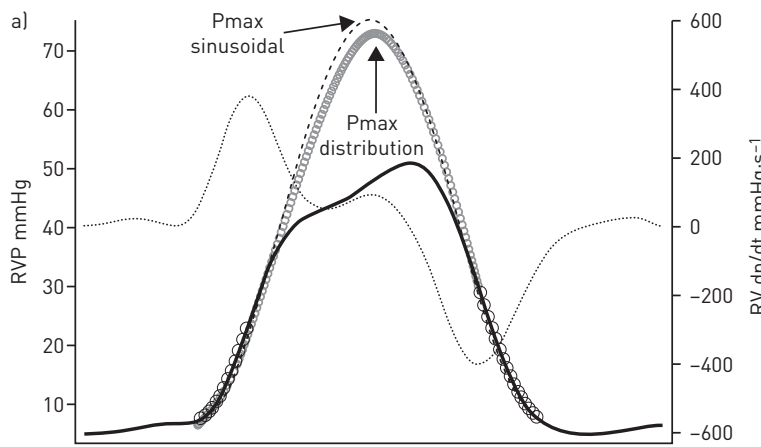

C) Proof: $E F=\quad \frac{\text { Ees }}{\text { Ees }+E a}$

$$
\text { (Ees }+ \text { Ea) } E F=\text { Ees }
$$

$\left(\frac{E S P}{E S V}+\frac{E S P}{S V}\right) E F=\frac{E S P}{E S V}$

$\left(\frac{(E S V+S V) \cdot E S P}{E S V \cdot S V}\right) E F=\frac{E S P}{E S V}$

$\left(\frac{E S V+S V}{E S V \cdot S V}\right) E F=\frac{1}{E S V}$

$\left(\frac{E S V+S V}{S V}\right)=\frac{1}{E F}$

$\left(\frac{E S V+S V}{S V}\right)=\left(\frac{E S V+S V}{S V}\right) \vee$

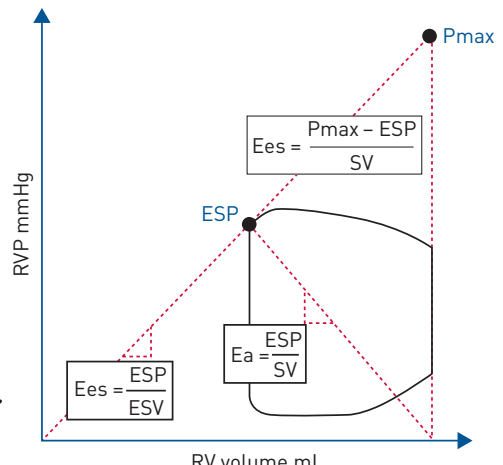

b)
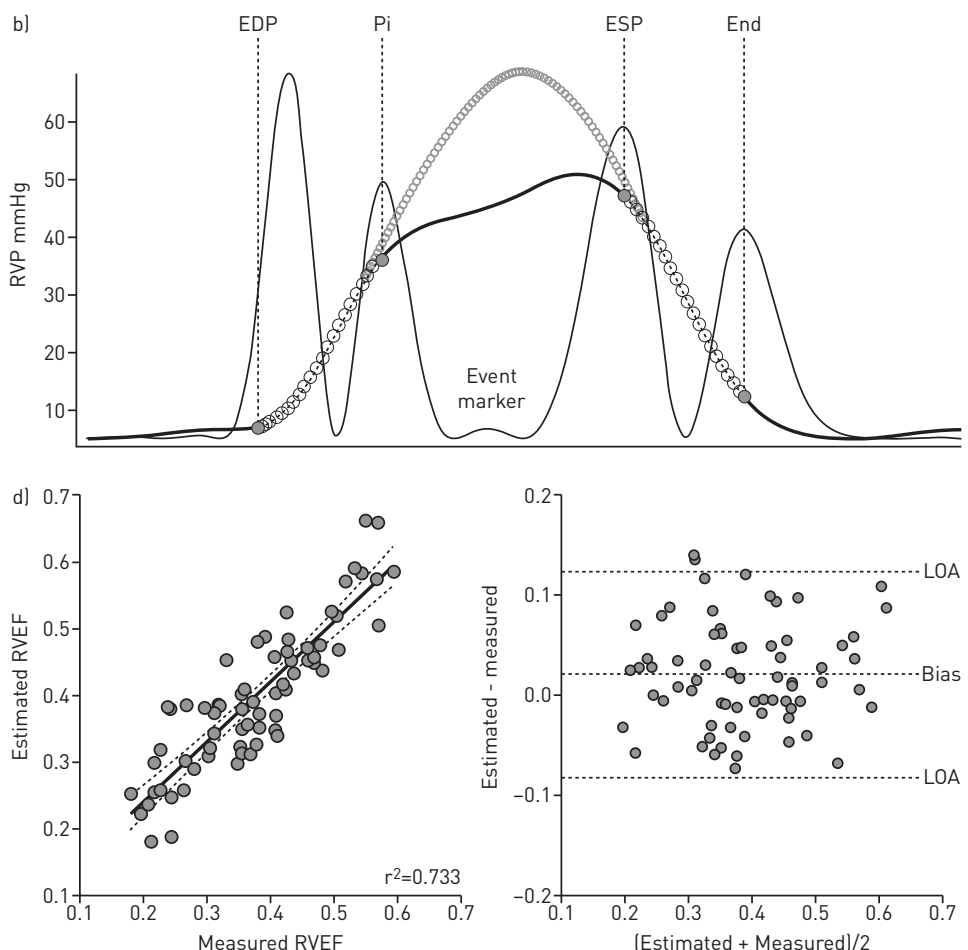

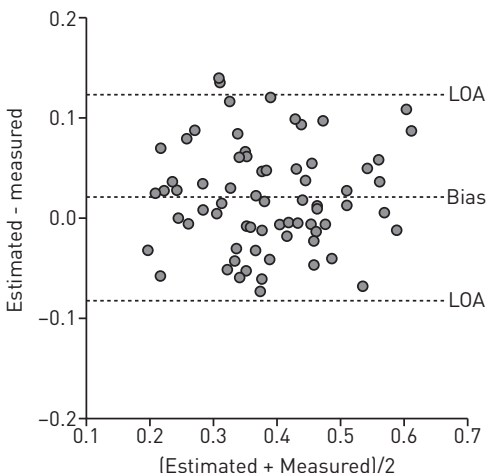

FIGURE 1 a) Comparison of Pmax (the theoretical pressure generated within the right ventricle (RV) if contraction remained isovolumic) predicted from laboratory data by application of conventional sinusoidal (hatched line) and Weibull distribution (solid grey line) functions using the same right ventricular pressure (RVP) segments (open circles superimposed on the solid black line depicting the RVP waveform). On average, Pmax values predicted by the distribution function were $3 \pm 7 \mathrm{mmHg}$ lower than those predicted by the sinusoidal function. b) For right ventricular ejection fraction (RVEF) prediction, the signal average of a series of RVP waveforms was created (thick black line) and its second derivative squared to produce four upright peaks (thin black line labelled "event marker"). These peaks were then used to define the "up and down" pressure segments for Pmax prediction lopen circles and grey line, respectively) as the intervals from half of the first peak (end-diastolic pressure, EDP) to the second peak (the first inflection point, Pi), and from the third peak (end-systolic pressure, ESP) to the fourth. The third peak approximates the point of maximal time varying elastance with RVP at this point regarded as an estimate of true ESP. c) Proof of the relationship $\mathrm{EF}=\mathrm{Ees} /(\mathrm{Ees}+\mathrm{Ea})$ in which both sides of the equation are resolved to the identical term. d) Correlation between RVEF directly calculated from continuous volume measurements as stroke volume (SV)/end-diastolic volume and RVEF estimated from the RVP waveform along with the BlandAltman plot showing the mean difference between methods (bias) and the limits of agreement (LOA). EF: ejection fraction; ESV: end-systolic volume; Ees: end-systolic ventricular elastance; Ea: arterial elastance.

with and without adjustment for repeated measures and included scatter plots to describe correlation, Bland-Altman plots to define accuracy (bias) and precision (limits of agreement), and four-quadrant concordance testing to determine uniformity of directional change. Estimated data were considered potentially interchangeable with measured values when the bias was $\leqslant 10 \%$ of the mean of all measured values, overall error (standard deviation of the bias $\times 1.96 /$ the mean of all ejection fraction values) was $\leqslant 30 \%$, and concordance $\geqslant 90 \%$.

Finally, preliminary clinical comparison of RVEF predicted from RVP recorded during diagnostic right heart catheterisation and that measured on the same day by cMRI was performed in six patients, three with pulmonary arterial hypertension and three with heart failure with preserved ejection fraction.

Measured RVEF values ranged from 0.18 to 0.59 (mean \pm SD $0.38 \pm 0.11$ ), and estimated RVEF from 0.18 to $0.66(0.40 \pm 0.11)$. For all data, there was strong correlation $\left(\mathrm{r}^{2}=0.733, \mathrm{p}<0.0001\right)$ with a bias of $0.03(8 \%$ of mean) and limits of agreement from -0.9 to 0.13 (figure $1 \mathrm{~d}$ ). Overall error was $27 \%$, and concordance 92\% (plot not shown). When corrected for repeated measures (data not shown), correlation remained strong $\left(\mathrm{r}^{2}=0.919, \mathrm{p}<0.0001\right)$, bias was 0.02 ( $6 \%$ of mean) with limits of agreement from -0.03 to 0.07 , overall error declined to $12.5 \%$, and concordance improved to $100 \%$.

RVEF measured by cMRI in six patients ranged from 0.30 to 0.70 and estimated RVEF from 0.32 to 0.64 . The difference between estimated and cMRI-derived RVEF was $\leqslant 10 \%$ in all patients except for one with significant tricuspid regurgitation (estimated RVEF 0.38, cMRI RVEF 0.70). However, estimated RV end-diastolic volume index in this patient (calculated as SV index measured by indirect Fick/estimated ejection fraction) was virtually the same as that measured by cMRI $\left(94 \mathrm{~mL} \cdot \mathrm{m}^{-2}\right.$ versus $\left.88 \mathrm{~mL} \cdot \mathrm{m}^{-2}\right)$. This observation appears to reflect the fact that when measured by cMRI, RVEF represents both forward and regurgitant flow. 
Although RVEF and Ees/Ea derived from RVP waveforms and SV both represent the composite balance between contractility and afterload, defining a specific relationship between them is challenging. Results of this proof of concept study involving both experimental and clinical data support the hypothesis that an alternative method for analysing RVP waveforms can provide quantitative estimates of RVEF without measurement of RV volume.

The study is based on the premise that ejection fraction can be approximated within limits as Ees/(Ees $+\mathrm{Ea})$ (figure 1c) then simplified to 1-(ESP/Pmax), a function similar to that previously described as an index of RV-pulmonary arterial coupling [11]. While this relationship removes the need for SV measurement, it is dependent upon consistent values for Pmax and ESP. When compared to conventional methods for Pmax prediction, preliminary data suggest our alternative approach produces similar results. In contrast, relative to the common practice of using mean pulmonary artery pressure as a surrogate for ESP, our method defines ESP in a manner more consistent with the point of maximal RV elastance [10].

Results of the study need to be interpreted in the context of limitations. Most importantly, as with single beat estimates of Ees based upon Pmax, the method assumes $V_{0}$ of the end-systolic pressure volume relationship to be $0 \mathrm{~mL}$ [12], which is rarely true for either ventricle [13, 14]. While specific definition of how variation in $\mathrm{V}_{0}$ affects accuracy of pressure-based RVEF prediction remains to be determined, interventions known to affect $V_{0}$ in swine are reflected in the experimental dataset [15], and the small clinical sample includes patients with $\mathrm{PH}$, also shown be associated with a wide range of $\mathrm{V}_{0}$ [12]. Our study results indicate that despite variation in $V_{0}$, RVEF estimated by the pressure-based method reasonably approximated RVEF derived from direct measurements. These preliminary observations suggest that while a degree of $\mathrm{V}_{0}$-dependence is inherent to the method, the error imparted by variation in $\mathrm{V}_{0}$ may not be prohibitive.

In summary, this proof of concept study suggests that estimation of RVEF may be feasible without measurement of RV volume. When combined with SV measurement, this method can allow for quantifying Ees and Ea as individual variables and specifically defining how alterations in each affected an observed acute RVEF change during an intervention. These preliminary results support further validation studies.

Paul M. Heerdt $\circledast^{1}$, Vitaly Kheyfets ${ }^{2}$, Sofia Charania ${ }^{1}$, Ahmed Elassal $^{1}$ and Inderjit Singh ${ }^{3}$

${ }^{1}$ Dept of Anesthesiology, Division of Applied Hemodynamics, Yale School of Medicine, New Haven, CT, USA. ${ }^{2}$ Dept of Bioengineering, School of Medicine, University of Colorado Denver, Anschutz Medical Center, Aurora, CO, USA. ${ }^{3}$ Division of Pulmonary, Critical Care, and Sleep Medicine, Dept of Medicine, Yale School of Medicine, New Haven, CT, USA.

Correspondence: Paul M. Heerdt, 330 Cedar St, TMP 3, New Haven, CT 06520, USA. E-mail: paul.heerdt@yale.edu

Received: 16 Aug 2019 | Accepted after revision: 13 Nov 2019

Acknowledgements: The authors would like to thank Daniel Burkhoff for his insightful comments on fundamental principles of cardiac pressure-volume analysis.

Author contributions: P.M. Heerdt: data analysis, manuscript preparation; V. Kheyfets: data analysis, manuscript preparation; S. Charania: data analysis, manuscript preparation; A. Elassal: data analysis, manuscript preparation; I. Singh: manuscript preparation.

Conflict of interest: P.M. Heerdt is co-founder of the startup company RVMetrics, LLC, outside the submitted work. V. Kheyfets has nothing to disclose. S. Charania has nothing to disclose. A. Elassal has nothing to disclose. I. Singh has nothing to disclose.

\section{References}

1 Lahm T, Douglas IS, Archer SL, et al. Assessment of right ventricular function in the research setting: knowledge gaps and pathways forward. an official American Thoracic Society research statement. Am J Respir Crit Care Med 2018; 198: e15-e43.

2 Naeije R, Manes A. The right ventricle in pulmonary arterial hypertension. Eur Respir Rev 2014; 23: 476-487.

3 Vonk Noordegraaf A, Westerhof BE, Westerhof $\mathrm{N}$. The relationship between the right ventricle and its load in pulmonary hypertension. J Am Coll Cardiol 2017; 69: 236-243.

4 Tello K, Dalmer A, Axmann J, et al. Reserve of right ventricular-arterial coupling in the setting of chronic overload. Circ Heart Fail 2019; 12: e005512.

5 Tello K, Richter MJ, Axmann J, et al. More on single-beat estimation of right ventriculoarterial coupling in pulmonary arterial hypertension. Am J Respir Crit Care Med 2018; 198: 816-818.

6 Metkus TS, Mullin CJ, Grandin EW, et al. Heart rate dependence of the pulmonary resistancexcompliance (RC) time and impact on right ventricular load. PLoS One 2016; 11: e0166463.

7 Richter MJ, Peters D, Ghofrani HA, et al. Evaluation and prognostic relevance of right ventricular-arterial coupling in pulmonary hypertension. Am J Respir Crit Care Med 2020; 201: 116-119. 
8 Brewis MJ, Bellofiore A, Vanderpool RR, et al. Imaging right ventricular function to predict outcome in pulmonary arterial hypertension. Int J Cardiol 2016; 218: 206-211.

9 Bellofiore A, Vanderpool R, Brewis MJ, et al. A novel single-beat approach to assess right ventricular systolic function. J Appl Physiol 2018; 124: 283-290.

10 Sagawa K ML, Suga H, Sunagawa K. Cardiac Contraction and the Pressure-Volume Relationship. New York, Oxford University Press, 1988.

11 Vanderpool RR, Pinsky MR, Naeije R, et al. RV-pulmonary arterial coupling predicts outcome in patients referred for pulmonary hypertension. Heart 2015; 101: 37-43.

12 Trip P, Kind T, van de Veerdonk MC, et al. Accurate assessment of load-independent right ventricular systolic function in patients with pulmonary hypertension. J Heart Lung Transplant 2013; 32: 50-55.

13 Dell'Italia LJ, Walsh RA. Application of a time varying elastance model to right ventricular performance in man. Cardiovasc Res 1988; 22: 864-874.

14 Burkhoff D. Pressure-volume loops in clinical research: a contemporary view. J Am Coll Cardiol 2013; 62: 1173-1176.

15 Heerdt PM, Korfhagen S, Ezz H, et al. Accuracy of a simulation algorithm for modelling LV contractility, diastolic capacitance, and energetics using data available from common hemodynamic monitors and echocardiography. J Cardiothorac Vasc Anesth 2018; 32: 381-388. 